\title{
Hypochloraemia and Worse Clinical Outcome in Critically Ill Patients
}

\author{
Rainer Gatz ${ }^{1}$ and Paul Elbers ${ }^{2}$ \\ ${ }^{1}$ ICU, Herlev Hospital, 2730 Herlev, Denmark \\ ${ }^{2}$ ICU, Onze Lieve Vrouwe Gasthuis, Amsterdam, The Netherlands \\ Correspondence should be addressed to Rainer Gatz, rkh.gatz@t-online.de
}

Received 1 August 2012; Accepted 30 August 2012

Copyright (C) 2012 R. Gatz and P. Elbers. This is an open access article distributed under the Creative Commons Attribution License, which permits unrestricted use, distribution, and reproduction in any medium, provided the original work is properly cited.

Tani et al. recently found an association between hypochloraemia and worse clinical outcome in critically ill patients, albeit not as an independent risk factor [1].

The authors were unable to find any explanation for this association. From clinical experience we could invoke three possible causes: use of $\mathrm{NaHCO}_{3}$, use of loop diuretics, or renal compensation for acidosis, all of which might be more prevalent in sicker patients. One of the tables presents data about the "strong ion gap" (SIG) in the three subgroups of hyper-, normo-, and hypochloraemic patients, with mean values of, respectively, 3.5 versus 4.8 versus $6.2 \mathrm{mEq} / \mathrm{L}$. The corresponding $P$-value is $<0.0001$. The authors do not mention this in their discussion section, though. While the value of SIG as an independent risk factor for poorer clinical outcome may not be proven, there are a few studies that indicate such a relation [2-6]. The link between hypochloraemia and worse clinical outcome might thus be that hypochloraemia is an indicator of higher SIG values. It would be highly interesting if the authors could contribute to this aspect of their data.

\section{References}

[1] M. Tani, H. Morimatsu, F. Takatsu, and K. Morita, "The incidence and prognostic value of hypochloremia in critically ill patients," The Scientific World Journal, vol. 2012, Article ID 474185, 7 pages, 2012.

[2] A. T. Maciel and M. Park, "Differences in acid-base behavior between intensive care unit survivors and nonsurvivors using both a physicochemical and a standard base excess approach: a prospective, observational study," Journal of Critical Care, vol. 24, no. 4, pp. 477-483, 2009.
[3] C. Mann, B. Latal, B. Padden, I. Scheer, G. Goebel, and V. Bernet, "Acid-base parameters for predicting magnetic resonance imaging measures of neurologic outcome after perinatal hypoxia-ischemia: is the strong ion gap superior to base excess and lactate?" American Journal of Perinatology, vol. 29, no. 5, pp. 361-368, 2012.

[4] N. Balasubramanyan, P. L. Havens, and G. M. Hoffman, "Unmeasured anions identified by the Fencl-Stewart method predict mortality better than base excess, anion gap, and lactate in patients in the pediatric intensive care unit," Critical Care Medicine, vol. 27, no. 8, pp. 1577-1581, 1999.

[5] L. J. Kaplan and J. A. Kellum, "Initial pH, base deficit, lactate, anion gap, strong ion difference, and strong ion gap predict outcome from major vascular injury," Critical Care Medicine, vol. 32, no. 5, pp. 1120-1124, 2004.

[6] G. C. Funk, D. Doberer, F. Sterz et al., "The strong ion gap and outcome after cardiac arrest in patients treated with therapeutic hypothermia: a retrospective study," Intensive Care Medicine, vol. 35, no. 2, pp. 232-239, 2009. 


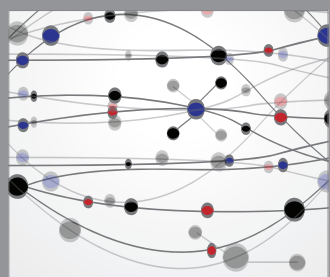

The Scientific World Journal
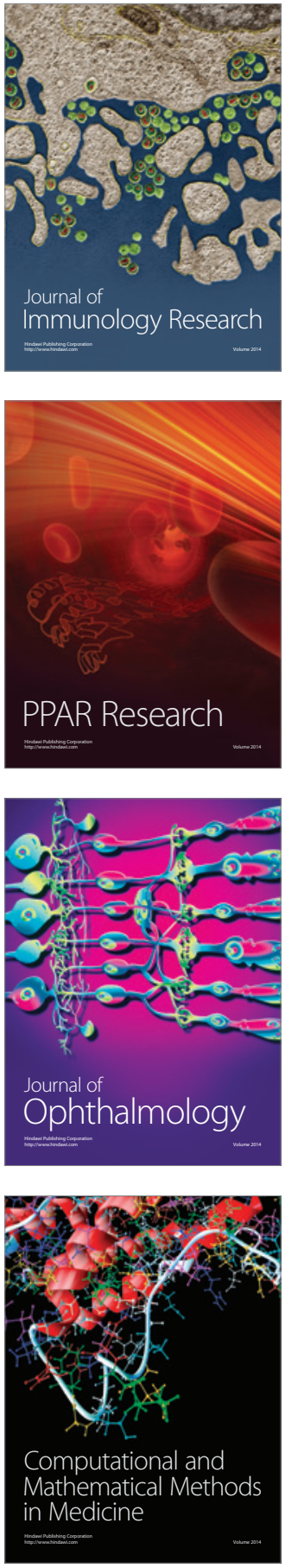

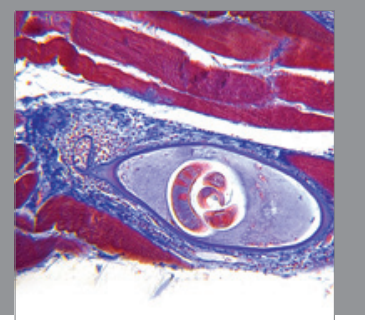

Gastroenterology

Research and Practice
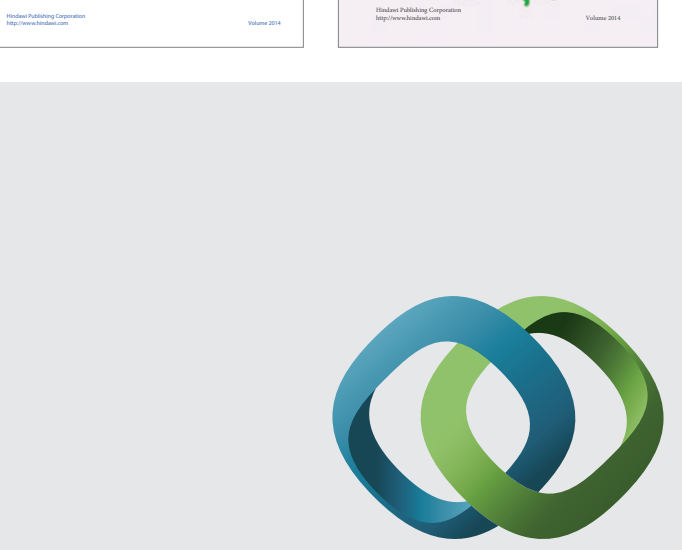

\section{Hindawi}

Submit your manuscripts at

http://www.hindawi.com
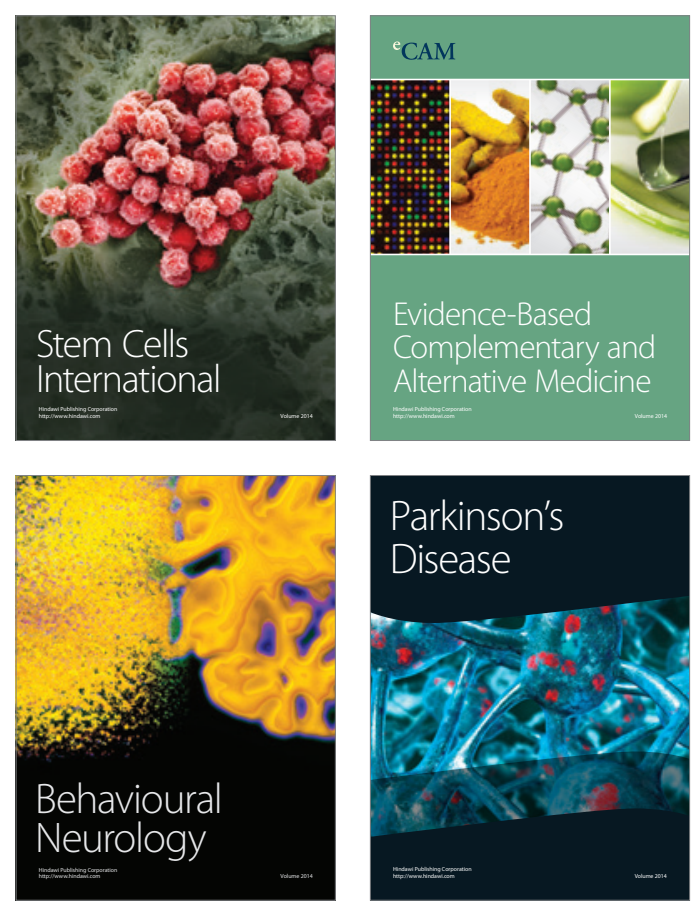

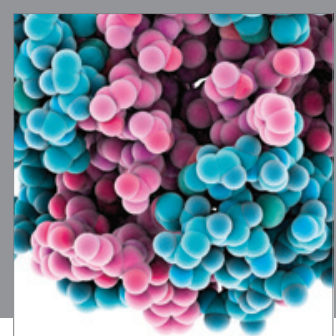

Journal of
Diabetes Research

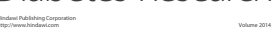

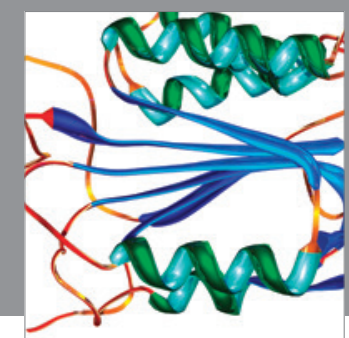

Disease Markers
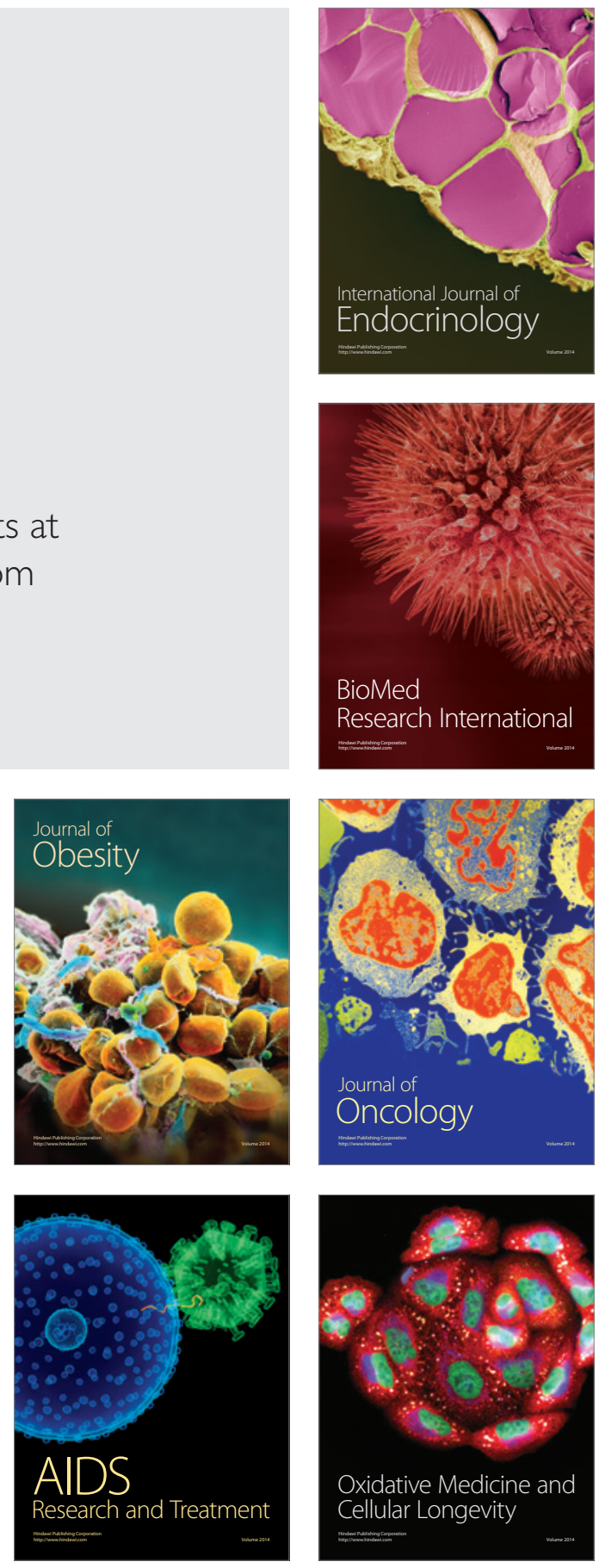